\title{
Comparative Analysis and Classification of Cassette Exons and Constitutive Exons
}

\author{
Ying Cui, ${ }^{1,2}$ Meng Cai, ${ }^{2,3}$ and H. Eugene Stanley ${ }^{2}$ \\ ${ }^{1}$ School of Mechano-Electronic Engineering, Xidian University, Xian 710071, China \\ ${ }^{2}$ Center for Polymer Studies and Department of Physics, Boston University, Boston, MA 02215, USA \\ ${ }^{3}$ School of Economics and Management, Xidian University, Xian 710071, China \\ Correspondence should be addressed to Meng Cai; mcai@bu.edu
}

Received 18 August 2017; Accepted 13 November 2017; Published 4 December 2017

Academic Editor: Yudong Cai

Copyright (C) 2017 Ying Cui et al. This is an open access article distributed under the Creative Commons Attribution License, which permits unrestricted use, distribution, and reproduction in any medium, provided the original work is properly cited.

\begin{abstract}
Alternative splicing (AS) is a major engine that drives proteome diversity in mammalian genomes and is a widespread cause of human hereditary diseases. More than $95 \%$ of genes in the human genome are alternatively spliced, and the most common type of AS is the cassette exon. Recent discoveries have demonstrated that the cassette exon plays an important role in genetic diseases. To discover the formation mechanism of cassette exon events, we statistically analyze cassette exons and find that cassette exon events are strongly influenced by individual exons that are smaller in size and that have a lower GC content, more codon terminations, and weaker splice sites. We propose an improved random-forest-based hybrid method of distinguishing cassette exons from constitutive exons. Our method achieves a high accuracy in classifying cassette exons and constitutive exons and is verified to outperform previous approaches. It is anticipated that this study will facilitate a better understanding of the underlying mechanisms in cassette exons.
\end{abstract}

\section{Introduction}

In most eukaryotic organisms, gene expression is regulated by alternative splicing (AS), which is the major posttranscriptional mechanism that promotes biological complexity. AS is a biological process in which one gene produces a variety of transcript isoforms through the distinct selection of splice sites during pre-mRNA splicing. AS plays an important regulatory role in proteome diversity $[1,2]$. Up to $95 \%$ of human genes are alternatively spliced in order to encode proteins with different functions [3], and approximately 15\% of human hereditary diseases and cancers are caused by AS [4].

Cassette exon splicing, also known as exon skipping, is the most prevalent form of alternative splicing in the human genome and accounts for 50 to 60 percent of all alternatively spliced events [5]. A cassette exon is a splicing event in which an intervening exon between two other exons from the mature mRNA sequence can be either included or skipped in order to generate two distinct protein isoforms. A number of recent discoveries have concluded that the cassette exon is closely associated with a broad range of human diseases
[6-11] such as renal cancer [9], neuromuscular diseases [10], and hearing loss [11]. Cassette exons have also been employed as a therapeutic strategy for producing the required proteins for genetic diseases $[10,12]$ such as the congenital myasthenia syndrome [12]. Despite its importance, because of its complexity, the cassette exon mechanism is not fully understood, and because of the limited availability of accurate computational tools, genome-wide detection of cassette exons remains a major challenge. Thus, we focus our attention here on a comparative analysis of the sequence features of cassette exons and constitutive exons in order to find the regulatory factors that contribute to cassette exon events. We also aim to construct a classification model that can distinguish between cassette exons and constitutive exons.

Various machine learning approaches are used in cassette exons identification [13-16]. Dror et al. used support vector machine (SVM), which is one of the classical machine learning methods, to classify cassette exons and constitutive exons conserved between human and mouse [13]. This research is highly dependent on conservation-based features, and thus its application on cassette exons detection is constrained 
to exons conserved between human and mouse genomes. $\mathrm{Su}$ et al. also proposed an SVM-based approach, which uses two classifiers [14]. The first classifier distinguishes authentic exons from pseudo exons, and the second classifier distinguishes cryptic exons from constitutive and skipped exons. This method did not use conservation information and therefore can be used more widely and easily. However, it only can achieve about $70 \%$ accuracy. Li et al. introduced an AdaBoost-based method to identify cassette exons and achieved $77.83 \%$ accuracy [15]. Recently, a method combining a gene expression programming (GEP) algorithm and a random forest (RF) model was proposed for identifying cassette exons in the human genome [16] and achieved a significantly higher accuracy $(90.87 \%)$ than previous studies. Although this hybrid program is a contribution that allows the investigation of cassette exons in human genes, its results still need improving. This GEP+RF method [16] also cannot speculate on the regulators of the formation mechanism of cassette exons events.

To understand why cassette exons occur and what kind of exon is more likely to be skipped, we here analyze and compare the sequence features of cassette exons with the constitutive exons in the human genome. We find that exons with shorter length, weaker splice sites, lower GC content, and more termination codons are more likely to be skipped during the splicing process. We propose a hybrid classification method based on an improved random forest model for distinguishing cassette exons in the human genome from constitutive exons. Computational simulation results indicate that our approach more accurately identifies cassette exons than previous methods.

\section{Materials and Methods}

2.1. Datasets. We use the HEXEvent database, which provides a list of human internal exons and reports all known splice events, as our source of alternative splicing information [17]. We downloaded the complete list of cassette exons and constitutive exons of the first human chromosome from the HEXEvent database. We also downloaded sequences of the first human chromosome from the UCSC Genome Browser [18] and extracted cassette exon and constitutive exon sequences using the lists we obtained from HEXEvent. Only exons without any other kind of AS events supported by the ESTs were used for analysis, and this produced a final dataset of 3120 cassette exons and 7316 constitutively spliced exons.

2.2. Feature Extraction and Analysis. Here, we compute and comparatively analyze the sequence features of cassette exons and constitutive exons to find out how they differ and to extract classification features for use in the next step. We compute 91 features including length, nucleotide composition (mononucleotide, dinucleotide, and trinucleotide), GC content, termination codon frequency, and splice site strength, and we use the $t$-test to analyze the differences between the two groups of data.

Although splice site strength signal is typically modeled using methods such as the first-order Markov model, the weight matrix model, and the maximum entropy (MaxEnt) model [19], the first-order Markov model disregards connections between nonadjacent positions and the weight matrix model is hindered by the hypothesis that different positions are independent. We thus use MaxEnt model to determine the strength of the splice site signal.

The MaxEnt model obtains the most likely distribution function of observables from statistical systems by maximizing the entropy under a set of constraints that model the incomplete information about the target distribution. The MaxEnt model consists of two distributions: the scilicet signal model $\left(P^{+}(x)\right)$ and the decoy probability distribution $\left(P^{-}(x)\right)$.

Let $p$ denote the unknown probability distribution. For a specific DNA sequence $x, p(x)$ represents its probability. Let $\widehat{p}$ be the approximation of $p$, where the entropy of $\widehat{p}$ is defined as

$$
H(\widehat{p})=-\sum \widehat{p}(x) \log _{2}(\widehat{p}(x)) .
$$

For a given sequence $x$, the signal strength of $x$ is

$$
L(X=x)=\frac{p^{+}(X=x)}{p^{-}(X=x)} .
$$

Here, $p^{+}(X=x)$ and $p^{-}(X=x)$ are the probability of $x$ from the distribution of signals $(+)$ and decoys $(-)$, respectively.

Table 1 and Figure 1 show the feature analysis results. We find that cassette exons are usually shorter in size and have a lower GC content, more termination codons, and weaker splice sites than constitutive exons. The strength of a splice site determines the skipping level in AS, so weak splice sites are suboptimal for the splicing mechanism. Apparently, short length, abundance of terminal codons, and weak splice signal hinder the ability of the splicing mechanism to recognize these exons, and the result is exon skipping.

2.3. Classifiers. We employ five binary classifiers to distinguish cassette exons from constitutive exons based on the 91 extracted features.

2.3.1. KNN Classifier. The $k$-nearest neighbors (KNN) algorithm is a linear pattern recognition method used for classification and regression [20]. For classification, KNN compares a test tuple and a collection of labeled examples in a training set. Each new sample in the prediction set is classified according to the class of the majority of its $k$-nearest neighbors in the training set. Parameter " $K$ " is the number of neighbors used to determine the class, and it strongly influences the identification rate of the KNN model.

2.3.2. SVM Classifier. The support vector machine (SVM) is a supervised machine learning algorithm based on statistical learning theory [21]. SVM is primarily used to solve classification and regression problems and has been successfully applied to bioinformatics tasks such as alternative splice sites identification and diagnostic method of diabetes [22, 23]. SVM uses a nonlinear mapping function to map original data into a high-dimensional feature space. It then constructs a hyperplane to be the surface that discriminates between positive and negative data. 
TABLE 1: Results of sequence feature analysis.

\begin{tabular}{lccc}
\hline Feature & Mean value of cassette exons & Mean value of constitutive exons & $p$ value \\
\hline Length & 142.85 & 176.39 & $<2.2 e-16$ \\
GC content & 0.4975 & 0.5062 & 0.009886 \\
Termination codon & 0.0128 & 0.0117 & 0.0006005 \\
$5^{\prime}$ splice strength & -13.43874 & -12.1145 & 0.0005285 \\
$3^{\prime}$ splice strength & -19.91228 & -18.29848 & 0.0002665 \\
\hline
\end{tabular}
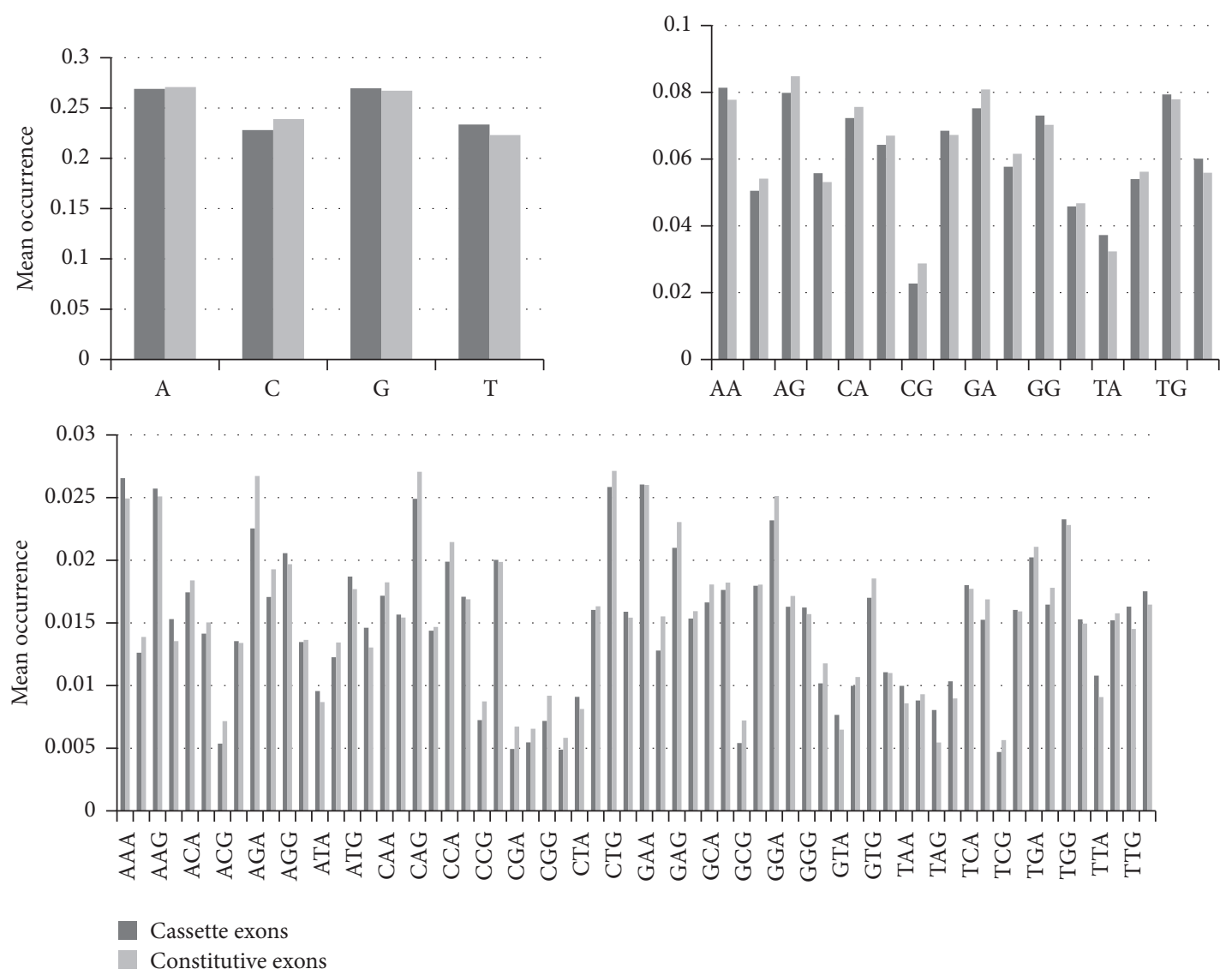

FIGURE 1: Mean occurrence of mononucleotide, dinucleotide, and trinucleotide.

2.3.3. RF Classifier. The random forest (RF) algorithm is an ensemble machine learning method developed by Breiman [24]. It has been widely applied to prediction problems in bioinformatics [25-27]. The RF algorithm consists of multibase tree-structured classifiers such as CART (classification and regression tree), and it is robust to noise, is not hindered by overfitting, and is computationally feasible. By applying CART as a base classifier, RF collects the outputs of all decision trees, tallies the result, and uses the result to classify the sample.

2.3.4. CF Classifier. The majority-voting rule in the traditional RF algorithm can cause minority categories to be misclassified. Thus, an improved RF function, the CForest (CF) function, has been proposed [28]. Unlike the standard $\mathrm{RF}$ algorithm based on the CART with its unfair splitting criterion, the CForest function uses a conditional inference framework to create an unbiased tree base classification model.

In the CForest algorithm, conditional inference trees are used to evaluate the association between the predictor variable and the response. The CForest algorithm works as follows. (i) The algorithm tests the global null hypothesis of independence between any of the input variables and the response and continues until this hypothesis is accepted. If it is not, the input variable with the strongest connection to the response is selected. (ii) The selected predictor variable is split into two disjoint sets. (iii) Steps (i) and (ii) are repeated.

The most important part of a forest framework is the splitting objective function. In the traditional RF, the most common measures are the information gain and the Gini impurity, which are biased towards relevant predictor variables. In response, Strobl et al. [29] proposed adding a conditional permutation importance scheme to the CForest 
framework. This permutation importance scheme uses a fitted forest model to partition the entire feature space, and it can be used to demonstrate the influence of a variable and to compute the importance of its permutation, conditional on all types of correlated covariates.

The CForest framework provides unbiased variable importance measurements and uses conditional permutation for feature selection. Here, the importance of a predictor variable is measured by comparing the accuracy of the prediction before and after the permutation.

Let $\bar{B}^{(t)}$ represent the out-of-bag (oob) sample for a tree $t$, with $t \in\{1, \ldots$, ntree $\}$. Here, ntree is the number of trees in the forest. The oob-prediction accuracy in tree $t$ before the permutation is

$$
\frac{\sum_{i} \bar{B}^{(t)} I\left(y_{i}=\widehat{y}_{i}^{(t)}\right)}{\left|\bar{B}^{(t)}\right|},
$$

where $\widehat{y}_{i}^{(t)}=f^{(t)}\left(x_{i}\right)$ is the predicted class for observation $i$ before permutation.

After permuting the value of $X_{j}$, the new accuracy is

$$
\frac{\sum_{i} \bar{B}^{(t)} I\left(y_{i}=\hat{y}_{i, \pi_{j} \mid Z}^{(t)}\right)}{\left|\bar{B}^{(t)}\right|}
$$

where $Z$ is the remaining predictor variables $Z=X_{1}, \ldots$, $X_{j-1}, X_{j+1}, \ldots$

Then, the variable importance of $X_{j}$ in tree $t$ can be expressed:

$$
\begin{aligned}
\mathrm{VI}^{(t)}\left(X_{j}\right)= & \frac{\sum_{i} \bar{B}^{(t)} I\left(y_{i}=\widehat{y}_{i}^{(t)}\right)}{\left|\bar{B}^{(t)}\right|} \\
& -\frac{\sum_{i} \bar{B}^{(t)} I\left(y_{i}=\widehat{y}_{i, \pi_{j} \mid Z}^{(t)}\right)}{\left|\bar{B}^{(t)}\right|} .
\end{aligned}
$$

Finally, the importance of each variable $X_{j}$ for the forest is calculated as an average over all trees:

$$
\mathrm{VI}\left(X_{j}\right)=\frac{\sum_{t=1}^{n \text { tree }} \mathrm{VI}^{(t)}\left(X_{j}\right)}{n \text { tree }}
$$

2.3.5. XGBoost Classifier. EXtreme Gradient Boosting (XGBoost) [30] is a scalable machine learning system for tree boosting and is one of the most popular machine learning methods in recent years. Based on the principle of gradient boosting machines proposed by Friedman [31], XGBoost produces a boosting ensemble of weak classification trees by optimizing a differentiable loss function with gradient descent algorithm. XGBoost deals with overfitting and is computationally efficient.

2.4. Performance Assessment. The $k$-fold cross-validation technique is commonly used to measure the performance of a classifier as it overcomes the problem of overfitting. The $k$ fold cross-validation method does not use the entire dataset to train the model but randomly splits the data into $k$ equalsized smaller subsets. Of the $k$ subsets, a single subset is retained to be the validation data for testing the model, and the remaining $k-1$ subsets are used as training data. The cross-validation process is then repeated $k$ times (the folds), with each of the $k$ subsets used once as test data to assess the performance of the model. We can then use the average of $k$ results from the fold to assess the performance of the constructed model.

A receiver operating characteristic (ROC) curve is a plot of the performance of a binary classifier system that shows the true positive rate against the false positive rate for different cut points. We often use this plot to calculate the area under a curve (AUC) to measure the performance of a classification model. The larger the area under the curve (a perfect prediction will make AUC equal to 1), the better the predictive accuracy of the model.

The evaluation indicators we use to measure performance are sensitivity, specificity, and total accuracy:

$$
\begin{aligned}
S_{n} & =\frac{\mathrm{TP}}{\mathrm{TP}+\mathrm{FN}}, \\
S_{p} & =\frac{\mathrm{TN}}{\mathrm{TN}+\mathrm{FP}}, \\
\mathrm{TA} & =\frac{\mathrm{TP}+\mathrm{TN}}{\mathrm{TP}+\mathrm{TN}+\mathrm{FN}+\mathrm{FP}},
\end{aligned}
$$

where TP is the number of correctly recognized positives, $\mathrm{FN}$ is the number of positives recognized as negatives, FP signifies the number of negatives recognized as positives, and TN is the number of correctly recognized negatives. Positives are cassette exons and negatives are constitutive exons.

\section{Results}

3.1. Sample Preparation. The 91 extracted sequence features (listed in Table 2) are combined in tandem to create an input classifier vector. To eliminate the negative effects of magnitude difference in value between different features, we scale the feature values to a range of -1 and 1 . Cassette exons and constitutive exons are considered to be positive and negative samples, respectively. In the 10 -fold cross-validation, the whole dataset is randomly and equally divided into ten sets. Each set is used as a testing set, and the remaining nine are used for training. We use a total of ten testing sets, and each training set is nine times the size of its corresponding testing set.

3.2. Performance of Different Classifiers. To highlight the good performance in discrimination of cassette exons and constitutive exons according to sequence features, we attempted to compare the classification results from KNN, SVM, RF, CF, and XGBoost approaches in this work. In the conduction of classifier model, parameter tuning is essential for building a binary-class model with high predictive accuracy and stability. In each classifier, parameters were optimized according to the prediction performance they 
TABLE 2: List of extracted features.

\begin{tabular}{lc}
\hline Feature subset & Number of features \\
\hline Length & 1 \\
Mononucleotide & 4 \\
Dinucleotide & 16 \\
Trinucleotide & 64 \\
Termination codon & 3 \\
GC content & 1 \\
Splice site strength & 2 \\
Total & 91 \\
\hline
\end{tabular}

eventually achieve. A parameter will be settled when it reaches the best prediction performance.

In the KNN model, we search the optimal parameter $K$ value by using a 10 -fold cross-validation. We estimate the predictive errors for a given set of $K$ values using crossvalidation and select the $K$ value that allows the subsequent KNN classification to yield optimal results. Thus, we optimize $20 K$ values $(K=1,2, \ldots, 20)$ using the 10 -fold crossvalidation. The KNN classifier achieves optimal performance when $K=7$.

In the SVM model, we examine the radial basis function (RBF) kernel, the linear kernel, the polynomial kernel, and the sigmoid kernel. Each kernel function has several parameters, and properly tuning them significantly affects the classification performance of the SVM model. We use a grid-search technique and 10-fold cross-validation to search the optimal parameter values of SVM using the four kernels. Table 3 supplies the predictive performance results of the four kernels in the SVM model. Of the four, the RBF kernel shows the best predictive accuracy, and we choose it to be the basic kernel function of the SVM classifier. There are two important parameters associated with RBF kernels: $C$ and $g$. We use a grid search and 10 -fold cross-validation and find the optimal $(C, g)$ pair to be $(25,0.000012)$.

In the random forest model, two parameters play an important role-ntree and mtry. The ntree parameter is the number of trees in the forest, and the mtry parameter is the number of variables available for splitting at each tree node. The forest error rate depends on two things: (i) the correlation between any two trees in the forest (increasing the correlation increases the error rate) and (ii) the strength of each individual tree in the forest (a tree with a low error rate is a strong classifier, and increasing the strength of the individual trees decreases the forest error rate). A larger number of trees produce a more stable model and covariate importance estimates but require more memory and a longer run time. Reducing mtry reduces both the correlation and the strength. Increasing it increases both. Somewhere in between is an "optimal" mtry range. We use a grid search and 10-fold cross-validation to find the optimal ntree and mtry. We find that the RF classifier performs best when ntree $=1000$ and mtry $=91$.

CForest parameter optimization is similar to the random forest. A grid search with 10 -fold cross-validation is used to

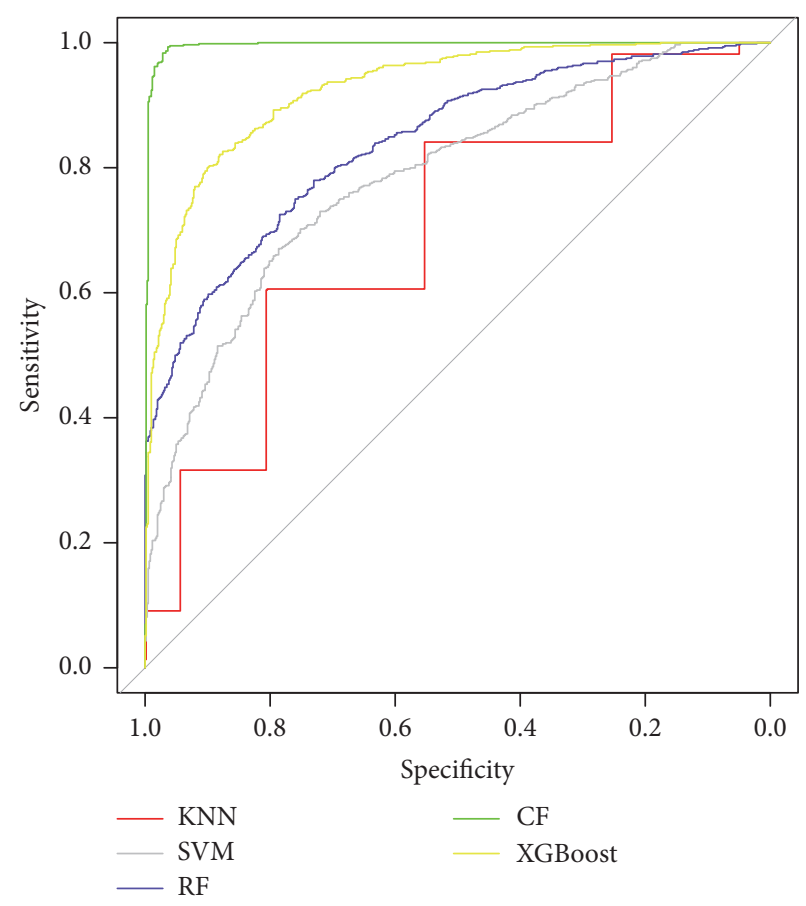

Figure 2: ROC curves of different classifiers.

determine the best ntree and mtry. The optimal (ntree, mtry) pair is $(1050,10)$.

In the XGBoost model, the type of model is set as treebased models. We mainly tuned two parameters, eta and max_depth. The eta is analogous to learning rate, which is the step size shrinkage used when getting the weights of new features after each boosting step to prevent overfitting. The max_depth is the maximum depth of a tree, which is used to control overfitting, as higher depth will make the model more complex. We use a grid search and 10-fold cross-validation to tune these two parameters and the optimal values are eta $=0.5$ and max_depth $=4$. Table 4 displays all the parameter optimization details of the KNN, SVM, RF, CF, and XGBoost models.

Using the optimal parameters, we construct five classifier models for identifying cassette exons based on KNN, SVM, RF, CF, and XGBoost models. We use sensitivity, specificity, total accuracy, and AUC to compare their predictive performances (shown in Table 5), and Figure 2 shows the ROC curves of different classifiers represented by different colors. It can be observed that CForest classifier significantly outperforms other classifiers. As another tree-based model, XGBoost wins the second place. We can see from Table 5 that there is an obvious imbalance between sensitivity and specificity in the RF classifier ( $\mathrm{Sn}=71.49 \%, \mathrm{Sp}=78.72 \%)$, where $\mathrm{Sn}$ is notably lower than $\mathrm{Sp}$, indicating that RF classifier can distinguish true constitutive exons from cassette exons but is not so effective in recognizing true cassette exons. This phenomenon can also be seen in KNN classifier $(\mathrm{Sn}=$ $67.03 \%, \mathrm{Sp}=75.57 \%$ ), another majority-voting principle based model. In contrast, CF classifier can do both; Sp is only 1.72 percent higher than $\mathrm{Sn}$. This is the case because 
TABLE 3: Classification results of SVM classifier with different kernels.

\begin{tabular}{|c|c|c|c|c|c|c|c|}
\hline \multirow{2}{*}{ Kernel } & \multicolumn{4}{|c|}{ Parameters } & \multirow{2}{*}{ Sensitivity (\%) } & \multirow{2}{*}{ Specificity (\%) } & \multirow{2}{*}{ TA (\%) } \\
\hline & C & $g$ & $d$ & $r$ & & & \\
\hline Linear & 1 & & & & 70.23 & 68.57 & 69.27 \\
\hline $\mathrm{RBF}$ & 25 & 0.000012 & & & 73.23 & 70.34 & 71.69 \\
\hline Poly & 14 & 0.015623 & 3 & & 72.26 & 70.12 & 71.08 \\
\hline Sigmoid & 357 & 0.000564 & & 0.3 & 73.27 & 69.78 & 71.44 \\
\hline
\end{tabular}

TABLe 4: Parameter optimization details in different classifiers.

\begin{tabular}{|c|c|c|c|c|}
\hline Classifier & Parameters & Step size in search & Search range & Optimal value \\
\hline KNN & $K$ & 1 & $1: 20$ & 7 \\
\hline \multirow{2}{*}{ SVM } & C & 1 & $1: 500$ & 25 \\
\hline & $g$ & 0.000001 & $10^{-6}: 1$ & 0.000012 \\
\hline \multirow{2}{*}{ Random forest } & ntree & 50 & $50: 2000$ & 1000 \\
\hline & mtry & 1 & $1: 91$ & 91 \\
\hline \multirow{2}{*}{ CForest } & ntree & 50 & $50: 2000$ & 1050 \\
\hline & mtry & 1 & $1: 91$ & 10 \\
\hline \multirow{2}{*}{ XGBoost } & eta & 0.1 & $0.1: 1$ & 0.5 \\
\hline & max_depth & 1 & $1: 10$ & 4 \\
\hline
\end{tabular}

TABLE 5: Classification performance of different classifiers.

\begin{tabular}{lcccc}
\hline Classifier & Sensitivity $(\%)$ & Specificity $(\%)$ & TA (\%) & AUC \\
\hline KNN & 67.03 & 75.57 & 70.45 & 0.7818 \\
SVM & 73.23 & 70.34 & 71.69 & 0.7865 \\
Random forest & 71.49 & 78.72 & 74.59 & 0.8411 \\
CForest & 95.90 & 97.62 & 96.69 & 0.9954 \\
XGBoost & 83.55 & 85.37 & 84.44 & 0.9270 \\
\hline
\end{tabular}

CForest classifier provides an unbiased measure of variable importance.

Generally, the nonlinear models (SVM, RF, CF, and XGBoost) are superior to the linear model (KNN), and the CForest model is the best. Theoretically, the nonlinear method performs better than the linear method when applied to self-learning and self-adjusting. The nonlinear model has a simpler structure and a higher classification performance. In a forest-based model, the splitting rule plays an essential role in the prediction accuracy. Traditional RF uses the majorityvoting rule to split, which shows a bias towards relevant predictor variables. This unfair splitting criterion tends to make the minority categories more likely to be misclassified. In response to this limitation, CForest is established by unbiased base classification trees based on a conditional inference framework. The CForest model assembles unbiased base classification trees into a conditional inference framework and thus eliminates the errors caused by the majority-voting rule used in the $\mathrm{KNN}, \mathrm{RF}$, and XGBoost models. In our case, there is a notable imbalance of the amount between cassette exons and constitutive exons. Thus, the CForest model is theoretically superior and exhibits a better predictive performance than the other classifiers.

3.3. Feature Importance. The CForest model provides an unbiased measure of variable importance, which can used to evaluate the importance of the features applied in the classification. We calculate the importance scores of all sequence features and rank the features according to their importance scores to explore the most essential features that predict cassette exons in our model. Figure 3(a) shows the top 15 features ranked according to their importance scores generated by the CForest classifier. The rank of a feature indicates its importance when discriminating cassette exons from constitutive exons. We can observe from Figure 3(a) that, in the top 15 features, 12 of them are dinucleotides and trinucleotides. Moreover, 8 of the 12 motifs ("gac," "agg," "acg," "tag," "ga," "cga," "aga," and "gaa") contain "ag" or "ga." This indicates that "ag" and "ga" play a role in the occurrence of cassette exon events. Additionally, it is speculated that length, $5^{\prime}$ splice site strength, and 3 splice site strength are the regulators of cassette exon events in the human genome. In Figure 3(b), we display the top 15 features ranked according to their importance scores generated in the random forest classification model. Random forest provides two measures to evaluate feature importance; here, we use the most common one known as Gini impurity. It can be seen from Figure 3(b) that, in the RF classifier, the most important feature is the length, which is the second important feature in CF classifier. Similar to CF, 6 of the top 15 features motifs ("ag," "gga," "gac," "aga," "gaa," and "ga") contain "ag" or "ga." Compared to CF, the difference of importance score in RF among the top 15 features is obviously smaller. This indicates that CF classifier can better reveal the distinct importance of different features in the process of classification, which might be a reason why CF exceeds RF in the classification of cassette exons and constitutive exons.

3.4. Comparison with Existing Methods. To demonstrate the superiority of our work, we compare the performance (sensitivity, specificity, and total accuracy) of our method to the methods proposed in [13-16]. Figure 4 shows the 


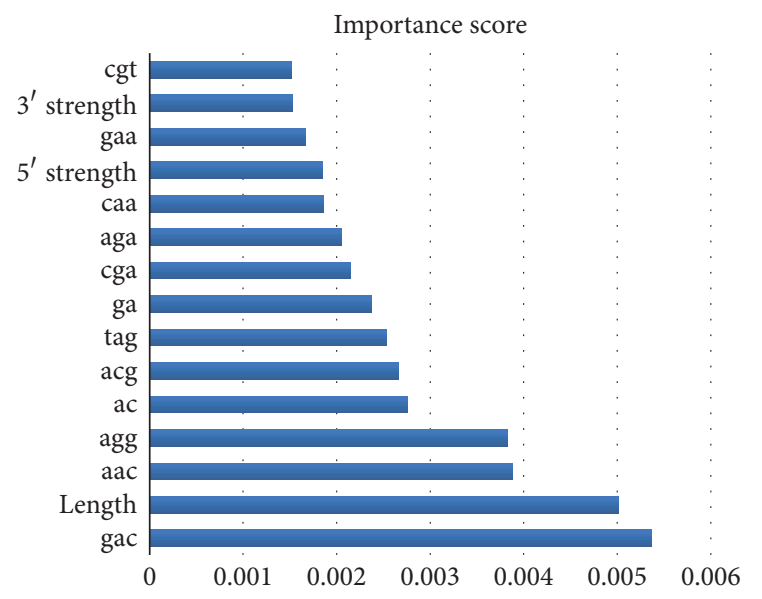

(a)

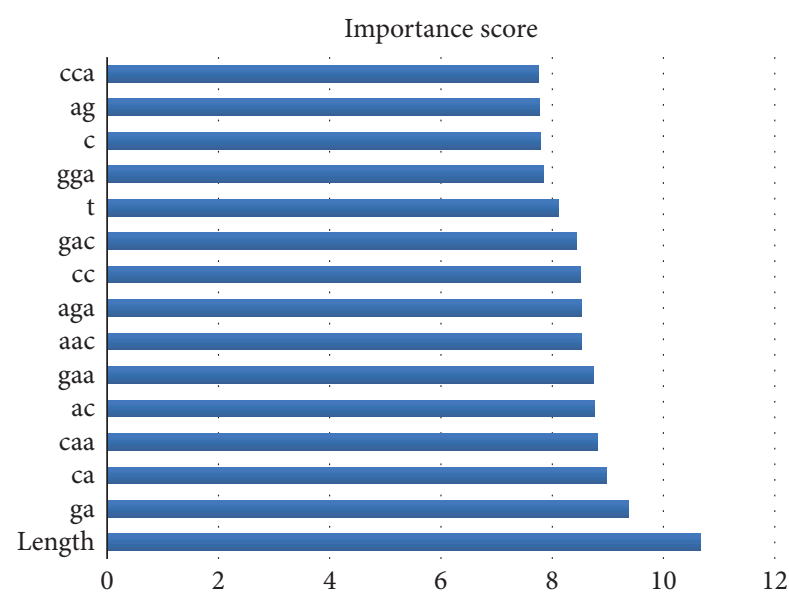

(b)

FIGURE 3: Importance rank of features (top 15) in different classification models. (a) CForest, (b) random forest.

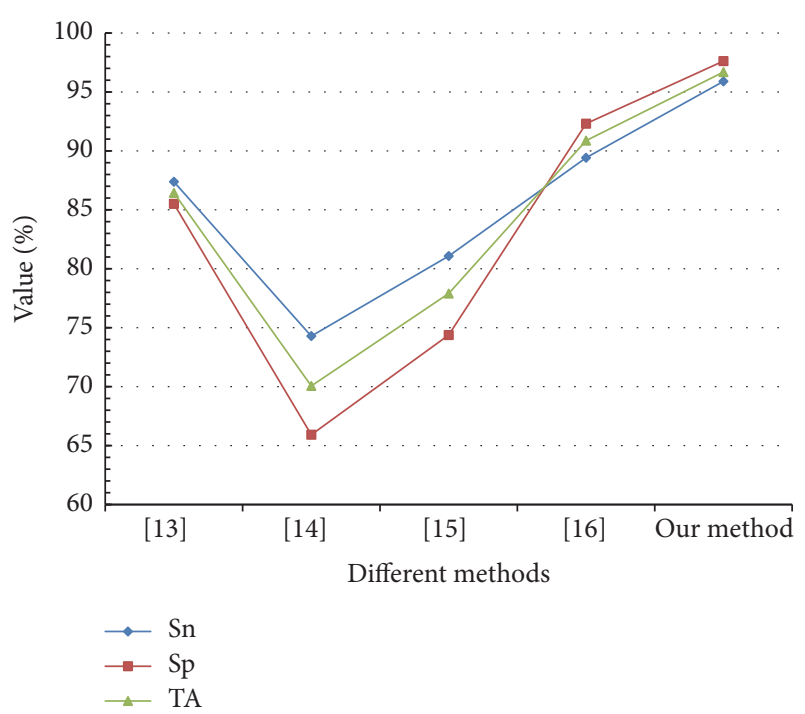

FIGURE 4: Performance comparison between existing methods and our method.

classification accuracy of different methods measured by Sn, $\mathrm{Sp}$, and TA. Our method achieved 95.90 percent sensitivity and 97.62 percent specificity, a higher level of accuracy than the other methods.

\section{Discussion}

In this paper, we used a comparative sequence feature analysis that includes length, nucleotide composition (mononucleotide, dinucleotide, and trinucleotide), GC content, termination codon frequency, and splice site strength to distinguish between cassette exons and constitutive exons. The results clearly indicate that cassette exons, the most common AS form of human, have shorter introns, a lower GC content, more termination codons, and weaker splice sites than constitutive exons.
These sequence features are combined in tandem to serve as an input of classifier. We attempted five different classifiers, that is, KNN, SVM, random forest, CForest, and XGBoost, to complement the discrimination task. We use grid search and 10-fold cross-validation to find the optimal parameters for every classification model, trying to make each of them achieve its best performance. Finally, the CForest classifier outperforms the other four models and reaches a total accuracy of $96.69 \%$. With the unbiased variable importance measure supplied in the CForest model, we ranked the importance of all features and displayed the top 15 of them, which is supposed to explore the regulator and contributor of cassette exon events in the human genome. In addition, we presented a comparison of the classification accuracy between our method and other existing methods to validate the superiority of our method in identifying cassette exons. It is obvious that our work provides an improved method for human cassette exons identification.

\section{Additional Points}

Data Availability Statement. Data used in this paper can be downloaded from http://hexevent.mmg.uci.edu/cgi-bin/HEXEvent/HEXEventWEB.cgi and http://hgdownload.soe.ucsc .edu/downloads.html, and the source code is available at https://sourceforge.net/projects/cassette-exon-prediction/files.

\section{Conflicts of Interest}

The authors declare that they have no conflicts of interest.

\section{Acknowledgments}

This work is supported by grants from the Natural Science Foundation of Shaanxi Province of China (no. 2016JQ6072), the National Natural Science Foundation of China (no. 71501153), Xian Science and Technology Program (no. XDKD001), and China Scholarship Council (201506965039, 201606965057). The Boston University Center for Polymer 
Studies is supported by NSF Grants PHY-1505000, CMMI1125290, and CHE-1213217, by DTRA Grant HDTRA1-14-10017, and by DOE Contract DE-AC07-05Id14517.

\section{References}

[1] E. Petrillo, M. A. Godoy Herz, A. Barta, M. Kalyna, and A. R. Kornblihtt, "Let there be light: regulation of gene expression in plants," RNA Biology, vol. 11, no. 10, pp. 1215-1220, 2014.

[2] K. D. Raczynska, A. Stepien, D. Kierzkowski et al., “The SERRATE protein is involved in alternative splicing in Arabidopsis thaliana," Nucleic Acids Research, vol. 42, no. 2, pp. 1224-1244, 2014.

[3] Q. Pan, O. Shai, L. J. Lee, B. J. Frey, and B. J. Blencowe, "Erratum: Deep surveying of alternative splicing complexity in the human transcriptome by high-throughput sequencing (Nature Genetics (2008) 40 (1413-1415))," Nature Genetics, vol. 41, no. 6, p. 762, 2009.

[4] Y. Marquez, J. W. S. Brown, C. G. Simpson, A. Barta, and M. Kalyna, "Transcriptome survey reveals increased complexity of the alternative splicing landscape in Arabidopsis," Genome Research, vol. 22, no. 6, pp. 1184-1195, 2012.

[5] H. Dvinge and R. K. Bradley, "Widespread intron retention diversifies most cancer transcriptomes," Genome Medicine, vol. 7, no. 1, article no. 45, 2015.

[6] S. Oltean and D. O. Bates, "Hallmarks of alternative splicing in cancer," Oncogene, vol. 33, no. 46, pp. 5311-5318, 2014.

[7] M. Danan-Gotthold, R. Golan-Gerstl, E. Eisenberg, K. Meir, R. Karni, and E. Y. Levanon, "Identification of recurrent regulated alternative splicing events across human solid tumors," Nucleic Acids Research, vol. 43, no. 10, pp. 5130-5144, 2015.

[8] S. C.-W. Lee and O. Abdel-Wahab, "Therapeutic targeting of splicing in cancer," Nature Medicine, vol. 22, no. 9, pp. 976-986, 2016.

[9] Y. Christinat, R. Pawłowski, and W. Krek, “JSplice: A high-performance method for accurate prediction of alternative splicing events and its application to large-scale renal cancer transcriptome data," Bioinformatics, vol. 32, no. 14, pp. 2111-2119, 2016.

[10] E. M. McNally and E. J. Wyatt, "Welcome to the splice age: Antisense oligonucleotide-mediated exon skipping gains wider applicability," The Journal of Clinical Investigation, vol. 126, no. 4, pp. 1236-1238, 2016.

[11] Y. Kim, H. R. Kim, J. Kim et al., "A novel synonymous mutation causing complete skipping of exon 16 in the SLC26A4 gene in a Korean family with hearing loss," Biochemical and Biophysical Research Communications, vol. 430, no. 3, pp. 1147-1150, 2013.

[12] S. Tei, H. T. Ishii, H. Mitsuhashi, and S. Ishiura, "Antisense oligonucleotide-mediated exon skipping of CHRNA1 premRNA as potential therapy for Congenital Myasthenic Syndromes," Biochemical and Biophysical Research Communications, vol. 461, no. 3, pp. 481-486, 2015.

[13] G. Dror, R. Sorek, and R. Shamir, "Accurate identification of alternatively spliced exons using support vector machine," Bioinformatics, vol. 21, no. 7, pp. 897-901, 2005.

[14] G. Su, Y.-F. Sun, and J. Li, "The identification of human cryptic exons based on SVM," in Proceedings of the 3rd International Conference on Bioinformatics and Biomedical Engineering, iCBBE 2009, China, June 2009.

[15] L. Li, Q. Peng, A. Shakoor, T. Zhong, S. Sun, and X. Wang, "A classification of alternatively spliced cassette exons using AdaBoost-based algorithm," in Proceedings of the 2014 IEEE
International Conference on Information and Automation, ICIA 2014, pp. 370-375, China, July 2014.

[16] X. Zhang, Q. Peng, L. Li, and X. Li, "Recognition of alternatively spliced cassette exons based on a hybrid model," Biochemical and Biophysical Research Communications, vol. 471, no. 3, pp. 368-372, 2016.

[17] A. Busch and K. J. Hertel, "HEXEvent: A database of human EXon splicing Events," Nucleic Acids Research, vol. 41, no. 1, pp. D118-D124, 2013.

[18] L. R. Meyer, A. S. Zweig, A. S. Hinrichs et al., "The UCSC Genome Browser Database: update 2006," Nucleic Acids Research, vol. 34, no. 90001, pp. D590-D598, 2006.

[19] G. Yeo and C. B. Burge, "Maximum entropy modeling of short sequence motifs with applications to RNA splicing signals," Journal of Computational Biology, vol. 11, no. 2-3, pp. 377-394, 2004.

[20] L. E. Peterson, “K-nearest neighbor," Scholarpedia, vol. 4, no. 2, article 1883, 2009.

[21] V. N. Vapnik, The Nature of Statistical Learning Theory, Springer, 2000.

[22] Y. Cui, J. Han, D. Zhong, and R. Liu, "A novel computational method for the identification of plant alternative splice sites," Biochemical and Biophysical Research Communications, vol. 431, no. 2, pp. 221-224, 2013.

[23] J. Zhang, J. Xu, X. Hu et al., "Diagnostic method of diabetes based on support vector machine and tongue images," BioMed Research International, vol. 2017, Article ID 7961494, 9 pages, 2017.

[24] L. Breiman, "Random forests," Machine Learning, vol. 45, no. 1, pp. 5-32, 2001.

[25] H. Kazan, "Modeling Gene Regulation in Liver Hepatocellular Carcinoma with Random Forests," BioMed Research International, vol. 2016, Article ID 1035945, 2016.

[26] X.-Y. Pan, Y.-N. Zhang, and H.-B. Shen, "Large-scale prediction of human protein-protein interactions from amino acid sequence based on latent topic features," Journal of Proteome Research, vol. 9, no. 10, pp. 4992-5001, 2010.

[27] X. Pan, L. Zhu, Y.-X. Fan, and J. Yan, "Predicting proteinRNA interaction amino acids using random forest based on submodularity subset selection," Computational Biology and Chemistry, vol. 53, pp. 324-330, 2014.

[28] T. Hothorn, K. Hornik, and A. Zeileis, "Unbiased recursive partitioning: a conditional inference framework," Journal of Computational and Graphical Statistics, vol. 15, no. 3, pp. 651674, 2006.

[29] C. Strobl, A.-L. Boulesteix, T. Kneib, T. Augustin, and A. Zeileis, "Conditional variable importance for random forests," $B M C$ Bioinformatics, vol. 9, no. 1, article 307, 2008.

[30] T. Chen and C. Guestrin, "XGBoost: a scalable tree boosting system," in Proceedings of the 22nd ACM SIGKDD International Conference on Knowledge Discovery and Data Mining, KDD 2016, pp. 785-794, August 2016.

[31] J. H. Friedman, "Greedy function approximation: a gradient boosting machine," The Annals of Statistics, vol. 29, no. 5, pp. 1189-1232, 2001. 

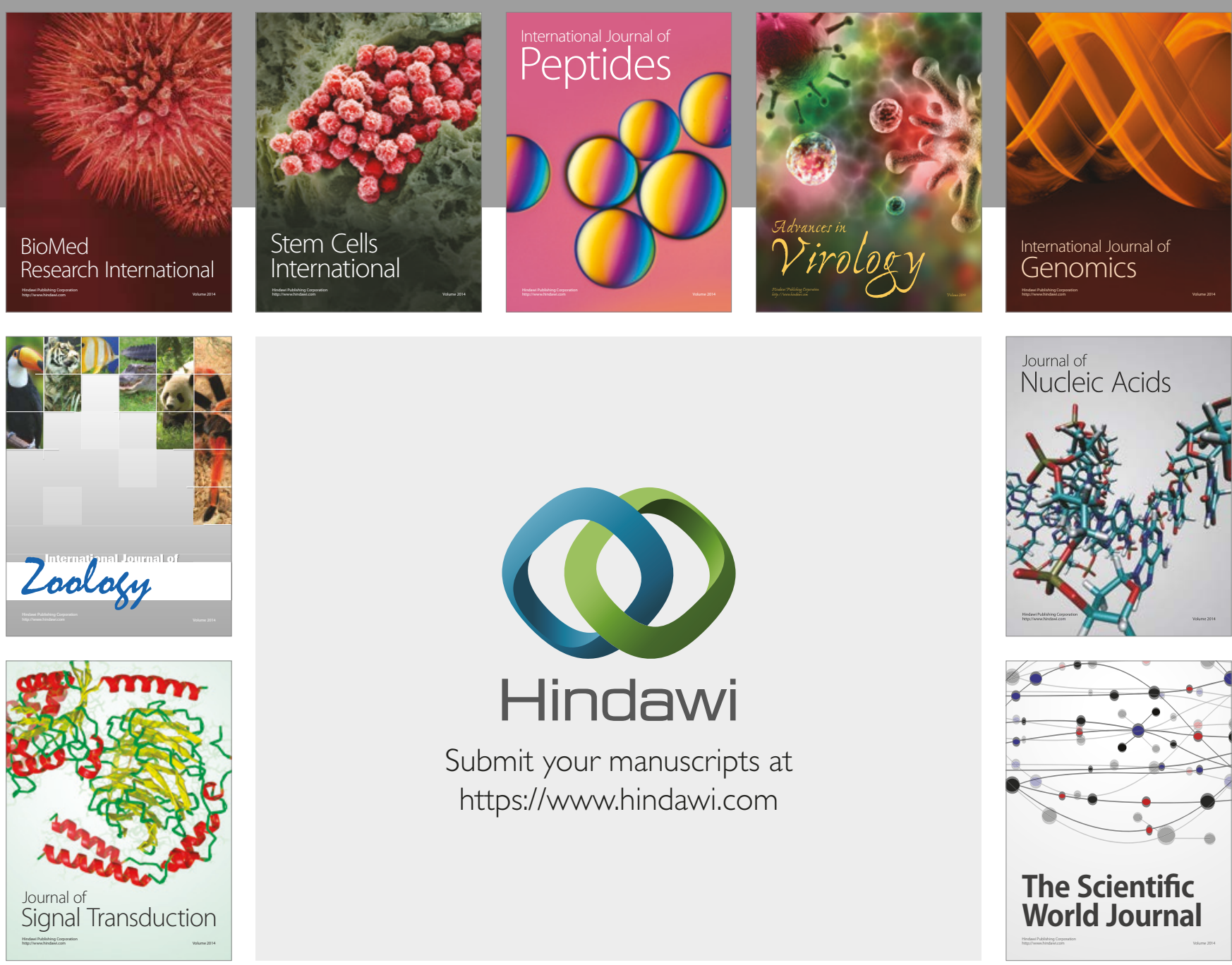

Submit your manuscripts at

https://www.hindawi.com
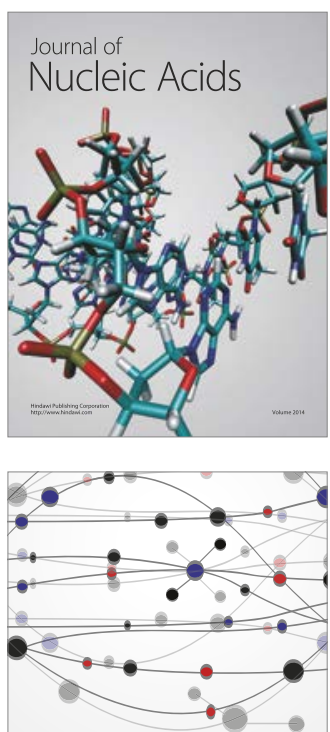

The Scientific World Journal

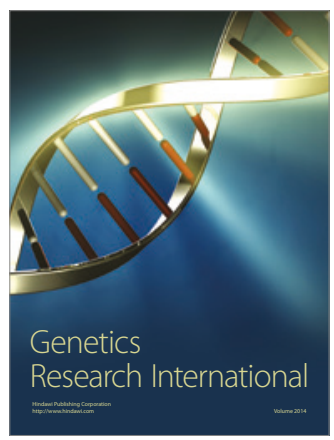

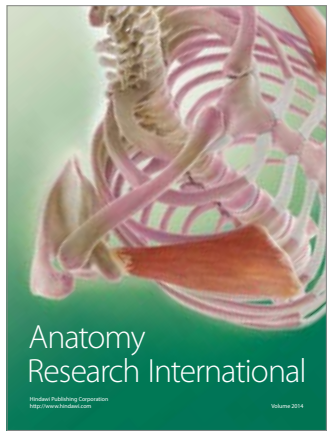

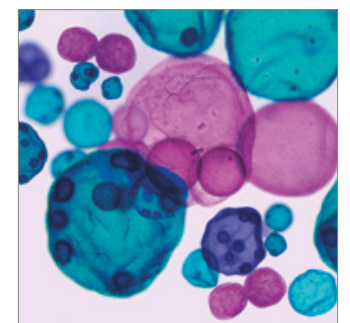

International Journal of Microbiology
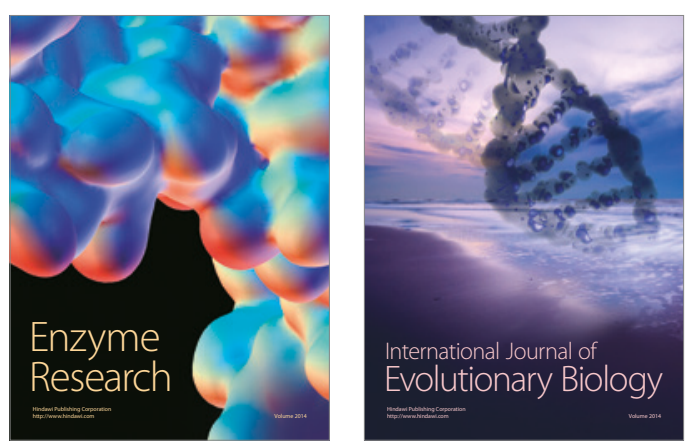
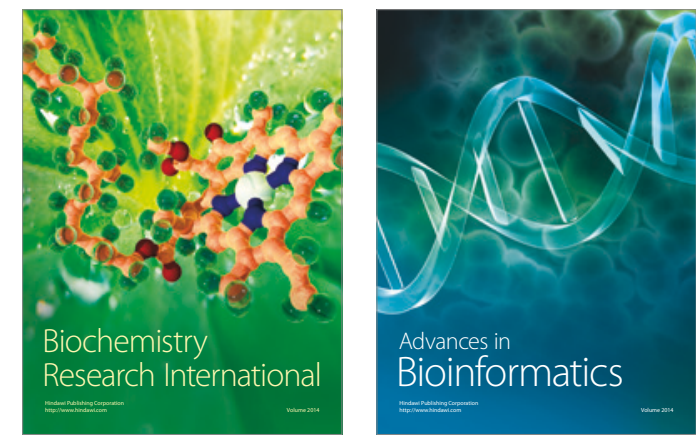

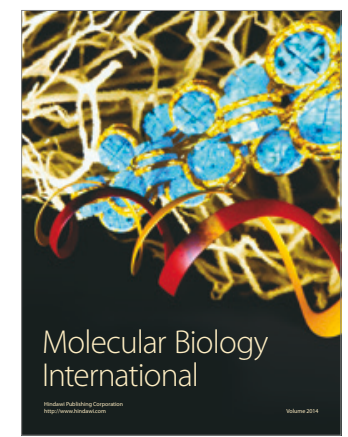

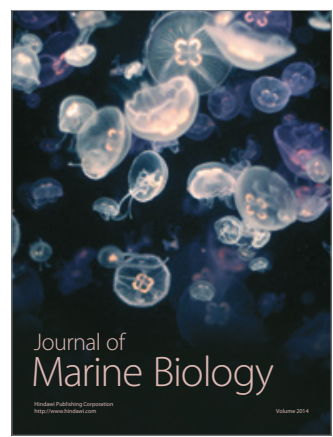

